\title{
Bile canalicular changes and defective bile secretion in Opisthorchis viverrini-infected hamsters
}

\author{
Lakhanawan Charoensuk ${ }^{1,6}$, Porntip Pinlaor ${ }^{2,6}$, Umawadee Laothong ${ }^{1,6}$, Puangrat Yongvanit ${ }^{3,6}$, \\ Chawalit Pairojkul $^{4,6}$, Yukifumi Nawa ${ }^{5}$ and Somchai Pinlaor ${ }^{1,6}$ \\ ${ }^{1}$ Department of Parasitology, Faculty of Medicine, Khon Kaen University, Thailand; \\ ${ }^{2}$ Centre for Research and Development in Medical Diagnostic Laboratory, Faculty of Associated Medical Sciences, Khon Kaen \\ University, Thailand; \\ ${ }^{3}$ Department of Biochemistry, Faculty of Medicine, Khon Kaen University, Thailand; \\ ${ }^{4}$ Department of Pathology, Faculty of Medicine, Khon Kaen University, Thailand; \\ ${ }^{5}$ Research Affairs, Faculty of Medicine, Khon Kaen University, Thailand; \\ ${ }^{6}$ Liver Fluke and Cholangiocarcinoma Research Center, Faculty of Medicine, Khon Kaen University, Thailand
}

\begin{abstract}
Infection with the liver fluke Opisthorchis viverrini (Digenea) (Poirier, 1886) causes bile duct injury and periductal fibrosis by chronic overproduction of inflammatory-mediators and eventually results in cholangiocarcinoma development. While extensive research works have been done on $O$. viverrini infection-associated changes of bile ducts and periductal fibrosis, little attention was paid on morphological and biochemical changes of the bile canaliculi (BC), the origin of bile flow. We aimed to investigate the morphological and functional alterations of $\mathrm{BC}$ in the liver of hamsters infected with $O$. viverrini at one and three months postinfection. Ultrastructural changes of $\mathrm{BC}$ showed dilatation of $\mathrm{BC}$ and significant reduction of the density of microvilli as early as at one month post-infection. Immunohistochemistry revealed that CD10, a BC marker, expression was reduced early as one month post-infection. The mRNA expression of the genes encoding molecules related to bile secretion including bile acid uptake transporters (slc10a1 and slcola1), bile acid dependent (abcb11) and independent (abcc2) bile flow and bile acid biosynthesis (cyp7a1 and cyp27a1) were significantly decreased at one month post-infection in association with the reduction of bile volume. In contrast, the expression of the mRNA of bile acid regulatory genes ( $f x r$ and $s h p-1)$ was significantly increased. These changes essentially persisted up to three months post-infection. In conclusion, $O$. viverrini infection induces morphological and functional changes of BC in association with the decrease of bile volume.
\end{abstract}

Keywords: liver fluke, gall bladder, morphological alterations, ultrastructure, bile secretion-related genes, bile volume, CD10

This article contains supporting information (Table S1) online at http://folia.paru.cas.cz/suppl/2014-61-512.pdf

Opisthorchiasis caused by a small human liver fluke, Opisthorchis viverrini (Poirier, 1886), after eating raw or undercooked fish infected with metacercariae, is still a major health problem in Southeast Asia, where 80 million people are estimated to be at risk for infection (IARC 2012). In heavy infections, which are approximately 5 to $10 \%$ of infected population, obstructive jaundice, cirrhosis, cholangitis, calculous cholecystitis or bile peritonitis might be observed and a portion of such cases will result in cholangiocarcinoma (CCA), the most serious complication of $O$. viverrini infection (IARC 2012).

Bile contains large quantities of bile acids, cholesterol and other organic molecules. Bile is essential for intestinal digestion, absorption of lipids, elimination of environmental toxins, excretion of endogenous compounds and metabolic products such as cholesterol, bilirubin and steroid hormones (Trauner and Boyer 2003). The major pathway of the bile acid synthesis is initiated via a member of the cytochrome P450 family. The $7 \alpha$-hydroxylation of cholesterol (CYP7A1) enzyme is responsible for the classical (neutral) bile acid biosynthetic pathway, whereas the mitochondrial sterol 27-hydroxylase (CYP27A1) is responsible for alternative (acidic) bile acid biosynthetic pathway (Chiang 2009). In addition, bile acid synthesis, transport and absorption are regulated by farnesoid $\mathrm{X}$ receptor $(f x r)$ and small heterodimer partner (shp-1) (Alrefai and Gill 2007). 
After synthesis, hepatocytes secrete bile into a canaliculus, which is formed by two or three adjacent hepatocytes (Yamamoto et al. 1990) through bile acid-dependent bile flow regulators (Bile salt export pump, BSEP or ATP-binding cassette, sub-family B member 11, abcb11), and bile acid-independent bile flow regulators (Multidrug resistance-associated protein 2, MRP2 encoded by ATPbinding cassette subfamily $\mathrm{C}$ member 2, abcc2 gene) (Alrefai and Gill 2007, Geier et al. 2007). Defect of bile secretion (or secretory system) results in hepatobiliary disease, i.e. intrahepatic cholestasis (Yeh et al. 2010), one of the relative risk for CCA (Tyson and El-Serag 2011).

Chronic inflammation leads to fibrosis involving the hepatic parenchyma resulting in biliary tree changes, which can contribute to cirrhosis and malignancy (Tyson and El-Serag 2011, Eaton et al. 2013). Oxidative stress in the liver and bile duct is sometimes accompanied by primary sclerosing cholangitis and cholestasis (Sekine et al. 2006). The morphological and functional changes of bile canaliculi (BC) are well demonstrated in bile duct ligation-induced cholestasis in rats (Takakuwa et al. 2002). Although $O$. viverrini infection is well known to cause inflammation-mediated oxidative/nitrative stress resulting in chronic periductal fibrosis, which is a risk factor of CCA (Pinlaor et al. 2004, Prakobwong et al. 2009, Sripa et al. 2012), it is unclear whether $O$. viverrini infection can affect the morphology and function of $\mathrm{BC}$, an origin of bile secretion.

In the present study, we investigated the morphological alteration of $\mathrm{BC}$ and the expression pattern of $\mathrm{CD} 10$, a marker molecule of BC. In addition, we determined the expression of genes encoding peptides/proteins related to bile acid uptake, bile acid biosynthesis, bile acid-dependent and independent bile flow and bile acid regulation.

\section{MATERIALS AND MEDTHODS}

\section{Parasites}

Metacercariae of Opistorchis viverrini were isolated from naturally infected cyprinid fish after digestion with $0.25 \%$ pep$\sin$ in $1.5 \% \mathrm{HCl}$ (Wako Pure Chemical Industries, Osaka, Japan) in $0.85 \% \mathrm{NaCl}$ solution as previously described (Prakobwong et al. 2009). The cysts containing actively moving larvae were used for infection to hamsters.

\section{Experimental design}

Male golden hamsters (4-6 weeks) were obtained from the Animal Unit, Faculty of Medicine, Khon Kaen University, Khon Kaen, Thailand. They were housed under a conventional condition and fed stock murine diet (CP-SWT, Thailand) and water $a d$ libitum. The study was conducted strictly following the recommendations in the guideline for the Care and Use of Laboratory Animals of the National Research Council of Thailand. The Animal Ethics Committee of Khon Kaen University approved this study (AEKKU31/2556). Twenty-four hamsters were divided into 12 each of control and $O$. viverrini-infected groups. The hamsters of the latter group were infected with each 50 metacer- cariae of $O$. viverrini by gastric intubation. Six hamsters from each group were sacrificed by diethyl ether inhalation at one month and three months post-infection.

\section{Specimen collection}

Bile in the gall bladder was collected using a syringe and an auto-pipette to measure its volume after animal fasting for $48 \mathrm{~h}$. Liver tissues were excised, treated with TRIzol ${ }^{\circledR}$ reagent (Invitrogen, Carlsbad, CA, USA) for total RNA isolation and snap frozen in liquid nitrogen for western blot analysis. The residual liver tissue was fixed in $10 \%$ buffered formalin for immunohistochemical study and the other fixed in $2.5 \%$ glutaraldehyde in $0.1 \mathrm{M}$ phosphate buffer for ultrastructural analysis.

\section{Western blot}

The liver tissues $(100 \mathrm{mg})$ were homogenised in ice-cold extraction buffer as previously described (Prakobwong et al. 2009). Protein concentration was determined using a Bio-Rad protein assay kit (Bio-Rad, Hercules, CA, USA) based on the Bradford's method. Crude liver extracts ( $20 \mu \mathrm{g}$ protein) were separated on a $10 \%$ and $15 \%$ SDS-PAGE gel and transferred to a polyvinylidene difluoride membrane (Amersham Bioscience, Piscataway, NJ, USA). The membranes were incubated with rabbit polyclonal anti-CD10 or goat polyclonal anti-MRP2 antibody (each 1 : 500; Santa Cruz Biotechnology, Santa Cruz, CA, USA), or mouse monoclonal anti-actin antibody (1:1000; Abcam, Cambridge, MA, USA) for overnight at $4^{\circ} \mathrm{C}$. After washing, membranes were incubated for $1 \mathrm{~h}$ at $4{ }^{\circ} \mathrm{C}$ with secondary antibody. Immunoreactivity was visualised using enhanced chemiluminescence (Amersham ${ }^{\mathrm{TM}}$ ECL $^{\mathrm{TM}}$ Prime Western blotting detection reagent, GE Healthcare Biosciences Corp., Piscataway, NJ, USA). Relative band intensity was analysed using ImageQuant TL 7.0 software (non-linear Dynamics, Durham, NC, USA).

\section{Immunohistochemistry}

Tissue sections were deparaffinised and autoclaved for antigen retrieval. The sections were incubated with rabbit antiCD10 (1 : 50, Santa Cruz Biotechnology) at room temperature for overnight and incubated with horseradish peroxidase-conjugated goat anti-rabbit IgG antibody (Amersham Bioscience) at room temperature for $1 \mathrm{~h}$. The 3, 3'-diaminobenzidine solution was used as a chromogenic substrate. The CD10 staining patterns of bile canaliculi were graded based on the percent of positive/negative of ten areas examined from one sample ( $n=6$ liver samples) under high power field as following: negative, no CD10 positive staining; $1+,<10 \%$ of CD10 positive areas; $2+$, $10-50 \%$ of CD10 positive areas; $3+,>50 \%$ of $\mathrm{CD} 10$ positive areas (Lin et al. 2004). Non-parametric Mann-Whitney $U$ test was used to determine the graded score of CD10 expression levels between normal and infected groups. $P$ values lower than 0.05 were considered statistically significant.

\section{Electron microscopy}

Three pieces of liver tissues were collected from proximal, middle and peripheral areas of the median lobe per animal. Liver tissues were cut $\left(1 \mathrm{~mm}^{3}\right)$, fixed in $2.5 \%$ glutaraldehyde in $0.1 \mathrm{M}$ phosphate buffer and post-fixed in $1 \%$ osmium tetroxide $\left(\mathrm{OsO}_{4}\right)$. Liver samples were performed by scanning electron microscopy (SEM) and transmission electron microscopy (TEM) as previously described (Jattujan et al. 2013). For SEM, the width of the bile canaliculi was taken from five randomly se- 
Table 1. The width of bile canaliculi, the grade of microvilli density and the grade of bile canaliculi pattern of the liver of normal and Opisthorchis viverrini-infected hamsters.

\begin{tabular}{lcccccc}
\hline \multirow{2}{*}{ Experimental groups } & \multicolumn{2}{c}{$\begin{array}{c}\text { Width of BC by SEM } \\
(\mu \mathrm{m}, \text { mean } \pm \text { S.D. }\end{array}$} & \multicolumn{2}{c}{$\begin{array}{c}\text { Microvilli density by TEM } \\
\text { (grade, mean } \pm \text { S.D.) }\end{array}$} & \multicolumn{2}{c}{$\begin{array}{c}\text { BC pattern } \\
\text { (grade, mean } \pm \text { S.D.) }\end{array}$} \\
\cline { 2 - 8 } & one month & three months & one month & three months & one month & three months \\
\hline Normal & $0.70 \pm 0.14$ & $0.72 \pm 0.14$ & $0.25 \pm 0.50$ & $0.25 \pm 0.50$ & $2.60 \pm 0.58$ & $2.80 \pm 0.44$ \\
OV-infected & $0.98 \pm 0.24^{*}$ & $1.00 \pm 0.22^{*}$ & $1.50 \pm 0.55^{*}$ & $2.17 \pm 0.75^{*}$ & $1.20 \pm 0.45^{*}$ & $1.67 \pm 0.82^{*}$ \\
\hline
\end{tabular}

Data are derived from all parts of liver (proximal, middle and peripheral regions) and presented as the mean \pm S.D. Statistical significance of the width of bile canalicular lumen was determined using an independent $t$-test. Non-parametric Mann-Whitney $U$ test was used to determine the density of microvilli and the graded score of $\mathrm{BC}$ pattern. $* P<0.05$, compared to normal groups for each correspondence time points; $\mathrm{BC}-$ bile canaliculi; $\mathrm{OV}$ - Opisthorchis viverrini; $\mathrm{BC}$ pattern - grading of CD10 staining

lected regions in each part of the liver sample, and the data were analysed using SMile View version 2.03. For TEM, the density of microvilli in the $\mathrm{BC}$ was evaluated by Digital Micrograph software (Gatan, Inc., Pleasanton, CA, USA) and graded based on five TEM electron micrographs in areas examined for each sample as following: grade 0 , densely packed microvilli; grade $1+, 25 \%$ loss of microvilli density; grade $2+, 50 \%$ loss of microvilli density; grade $3+, 75 \%$ or higher loss of microvilli density. Statistical significance of the width of bile canalicular lumen between normal and infected groups were determined using an independent $t$-test. Non-parametric Mann-Whitney $U$ test was used to determine the density of microvilli. $P$ values lower than 0.05 were considered statistically significant.

RNA isolation and quantification by real-time polymerase chain reaction

Total RNA was extracted from liver tissues using TRIzol ${ }^{\circledR}$ reagent (Invitrogen Corporation) and transcribed to cDNA with RevertAid H Minus M-MuLV Reverse Transcriptase (Fermentas, St. Leon-Rot, Germany). The primers used in this study were designed by Primer 3 software: abcc2 (5'-CCCTCACAAACTGCCTCTTC-3' and 5'-GGGTCGAGATTCATCCTCAG-3'); abcb11 (5'-CTAGGCTTGCTACAGATGCT-3' and 5'-CTGTACAGCTCCCGATAAAG-3'); slc10a1 (5'-GGTGCCCTACAAAGGCATTA-3' and 5'-GTTGCCCACATTGATGACAG-3'); slcola1 (5'-TGGACTTTTGTTGGGATCTT-3' and 5'-AAAAAGAAAAAGGGGATGCT-3'); cyp7a1 (5'-CACAAACTCCCTGTCATACC-3' and 5'-AGTGAACACAGAGCATCTCC-3'); cyp27a1 (5'-GTATCTGGCTACCTGCACTT-3' and 5'-CACACCAGTCACTTCCTTGT-3'); fxr (5'-ATCCTCTCTCCAGACAGACA-3' and 5'-CTCCAAGACATCAGCATCTC-3'); shp-1 (5'-CTCTCTTCCTGCTTGGGTTG-3' and 5'-GCTCAAGGCTCCAGAAAGAC-3') and gapdh (5'-AGAAGACTGTGGATGGCCCC-3' and 5'-TGACCTTGCCCACACCCTT-3').

Relative mRNA expression of the each sample was determined in duplicate by ABI7500 thermal cycler (Applied Biosystems, Foster City, CA, USA) using a FastStart Universal SYBR Green Master (ROX, Roche Applied Science, Mannheim, Germany). Samples were analysed in duplicates. Relative quantification of mRNA expression was determined using gapdh as an internal control, the data processed using the $2^{-\Delta \Delta \mathrm{Ct}}$ method (Prakobwong et al. 2009). The PCR amplicons were purified and sequenced by the First BASE Laboratories Sdn. Bhd. (ShahAlam, Selangor, Malaysia). Sequences of amplified fragment identities to hamster ( $100 \%$ for nucleotides and amino acids), or to rat, mouse and man over $90 \%$ for nucleotides and over $86 \%$ for amino acids are acceptable (Table S1).

\section{RESULTS}

\section{Ultrastructural changes of bile canaliculi (BC)}

\section{Scanning electron micrograph of hamster liver}

The ultrastructure of $\mathrm{BC}$ from the proximal, middle and peripheral areas of the median liver lobe showed similar results. Representative SEM images of proximal part of liver are shown in Fig. 1. The ultrastructure of BC in the control group is characterised by uniform and narrow lumen with numerous microvilli on the luminal surface. These findings were the same in the normal liver at one (Fig. 1A,B) and three months after experimental infection (Fig. 1E,F). In contrast, BC lumen was dilated in the infected groups at one month (Fig. 1C,D) and those changes persisted at three months (Fig. 1G,H). The average comparison of $\mathrm{BC}$ lumen width from the proximal, middle and peripheral parts of the liver between control and infected group is shown in Table 1.

\section{Transmission electron micrograph of hamster liver}

Representative TEM images of the proximal part of the liver are shown in Fig. 2. The TEM photographs of the normal liver showed abundant microvilli on the sinusoidal surface (Fig. 2A,C). As was expected from SEM observation, the density of microvilli in the canalicular lumen was significantly decreased at one month and the decrease persisted up to three months post-infection (Fig. 2B,D, Table 1).

\section{Changes of CD10 expression in the hamster liver}

To confirm ultrastructural changes triggered by $O$. viverrini infection, immunohistochemical staining pattern of CD10, a marker of BC, was examined (Fig. 3A-D). In the control group, CD10-immunoreactivity was seen as a 'chicken-wire'-like appearance, which is a typical normal staining pattern of the BC network (Watanabe et al. 1991). This staining pattern was maintained well in normal liver at three months. In the $O$. viverrini-infected group, CD10 expression was significantly reduced and a polygonal meshwork pattern disappeared at one and three months post-infection. The disappearance (or reduc- 

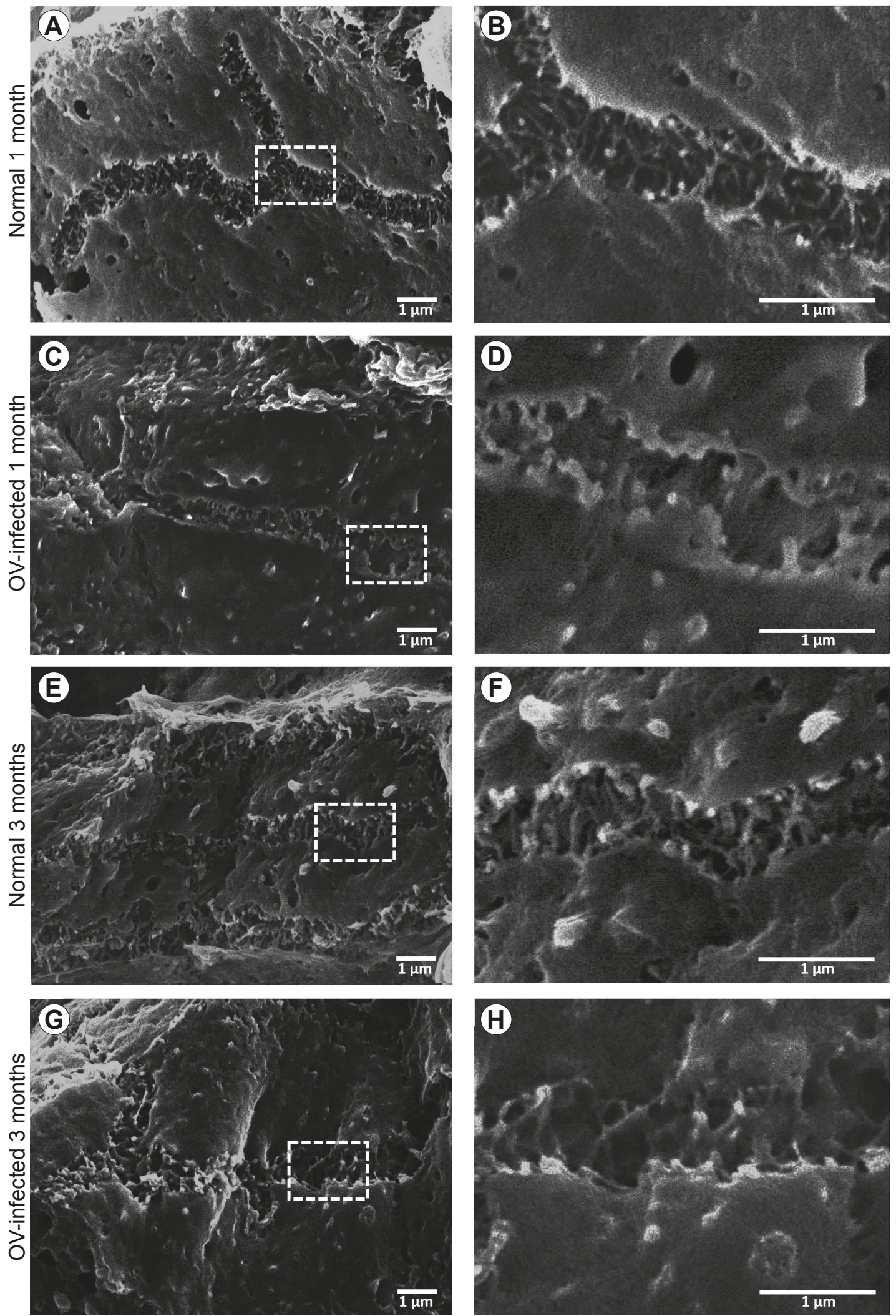

Fig. 1. Ultrastructural changes of bile canaliculi in hamster's liver observed by scanning electron microscopy. Representative SEM images of the proximal part of the median liver lobe showing bile canaliculi features of (A, E) normal hamsters and (C, G) Opisthorchis viverrini-infected hamsters at one and three months post-infection, respectively. A magnified view of bile canaliculi within the square is shown in $\mathbf{B}$ and $\mathbf{F}$ for normal and $\mathbf{D}$ and $\mathbf{H}$ for $O$. viverrini-infected hamster. Representative data are shown from three animals per groups. Abbreviations: OV - Opisthorchis viverrini. 

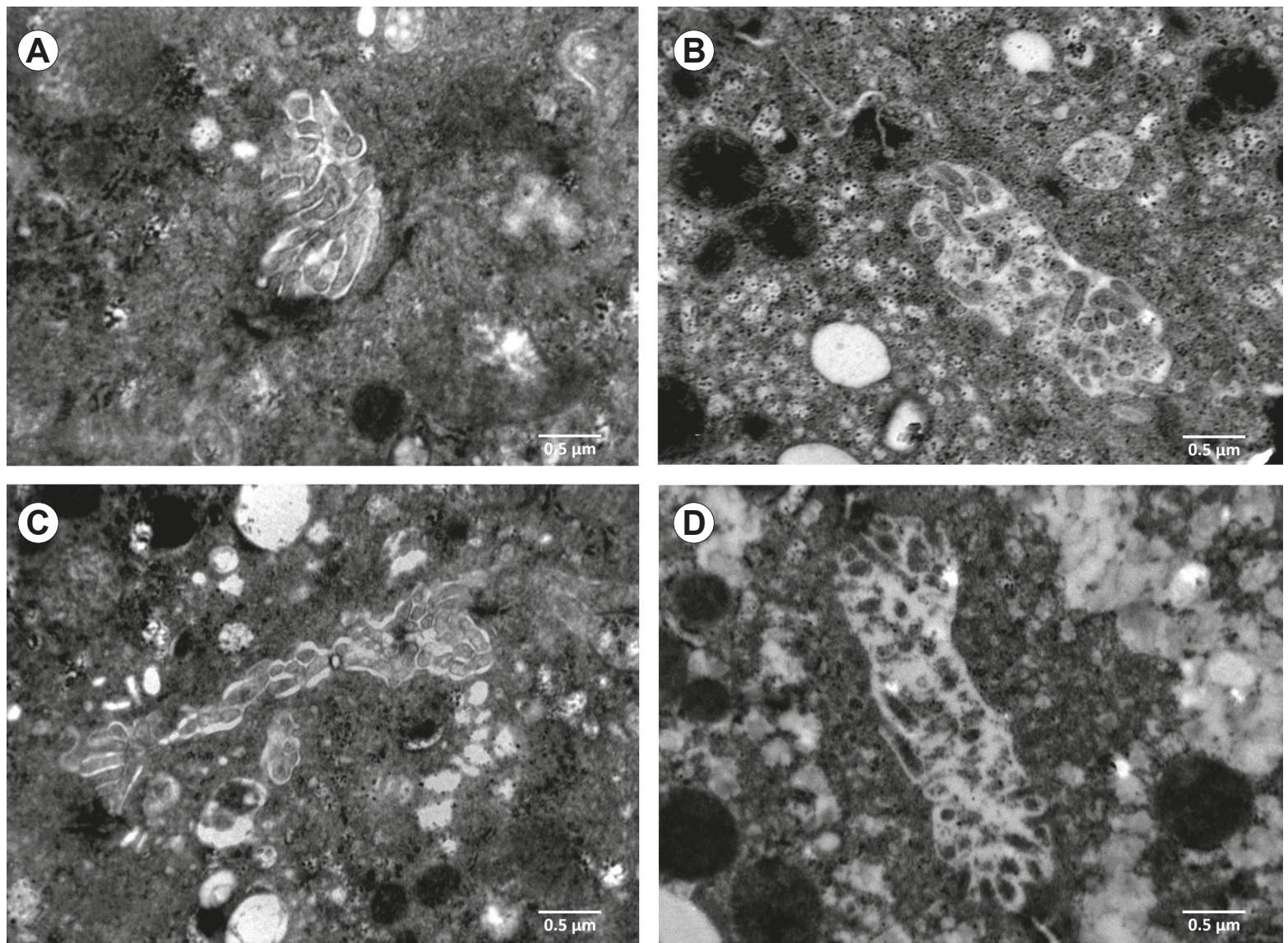

Fig. 2. Ultrastructural changes of bile canaliculi in hamster's liver observed by transmission electron microscopy. Representative TEM images of the proximal part of the median liver lobe showing bile canaliculi features of normal (A,C) and Opisthorchis viverrini-infected $(\mathbf{B}, \mathbf{D})$ livers at one $(\mathbf{A}, \mathbf{B})$ and three $(\mathbf{C}, \mathbf{D})$ months after experimental infection. Representative data are shown from three animals per groups.

tion) of CD10 was confirmed by semiquantitative measurement using western blot analysis (Fig. 3E,F).

\section{Decrease of bile volume and down-regulated expression of bile acid-dependent and independent bile flow related genes}

Bile volume in the gall bladder and the expression of the genes encoding protein involved in bile flow including bile acid-dependent bile flow $(a b c b 11)$ and bile acidindependent bile flow $(a b c c 2)$ are shown in Fig. 4. In the $O$. viverrini-infected group, bile volume significantly decreased at one and three months post-infection compared to that of the control (Fig. 4A). mRNA expression of abcb11 was significantly decreased at one month and still remained low level at three months post-infection (Fig. 4B), while abcc2 gene was significantly decreased at one and three months post-infection (Fig. 4C).

In relation to the $\mathrm{BC}$ function, we also evaluated the expression of $a b c c 2$ gene product (MRP2 protein), which is known as a bile acid-independent bile flow regulator, using western blot analysis. The results showed that the MRP2 protein level of $O$. viverrini-infected group was significantly lower than that of the control group at one and three months post-infection (Fig. 4D, 5; $P<0.05$ ).

Altered expressions of the genes involved in bile acid uptake transporters, bile acid biosynthesis and bile acid regulatory genes

We then investigated whether $O$. viverrini infection alters the mRNA expression of the genes encoding protein molecules involved in bile acid uptake transporters, bile acid biosynthesis and bile acid regulatory genes using real-time RT-PCR (Fig. 6). For this purpose, we selected slc10a1 (solute carrier family 10 member 1) and slcola1 (solute carrier organic anion transporter family, member $1 \alpha 1$ ) as bile acid uptake transporter genes, cyp $7 a 1$ and cyp27al as bile acid biosynthesis genes, and fxr and shp-1 genes as bile acid regulatory genes. In the $O$. viverriniinfected group at one and three months post-infection, the mRNA expression of slc10al and slcolal (Fig. 6A,B) and cyp7al and cyp27al (Fig. 6C,D; $P<0.05$ ) genes were significantly decreased compared to those of the control group. In contrast, the expression of $f x r$ and $s h p-1$ genes was significantly increased by $O$. viverrini infection (Fig. 6E,F). 

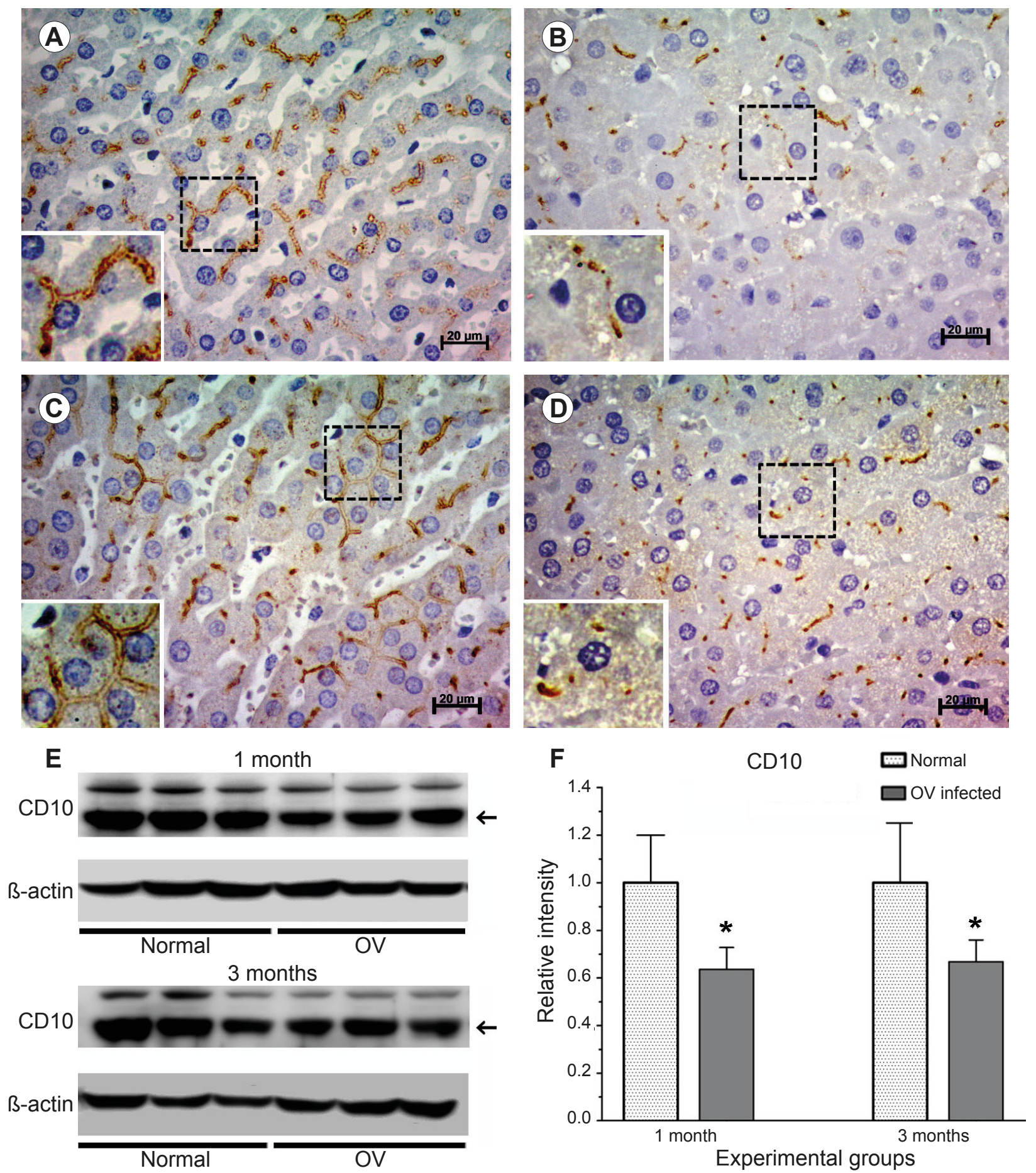

Fig. 3. Expression of bile canalicular characteristics in normal $(\mathbf{A}, \mathbf{C})$ and Opisthorchis viverrini-infected (B,D) hamsters; an immunohistochemical staining of CD10 at one $(\mathbf{A}, \mathbf{B})$ and three $(\mathbf{C}, \mathbf{D})$ months after experimental infection. The canalicular characteristics were assessed in 10 randomly selected fields $(\times 400)$ from six animals per group; magnified view is shown in inset images; $\mathbf{E}-$ protein expression levels of CD10 measured by western blot; F- showed relative intensity of band protein for CD10; data are mean \pm S.D. Statistical significance of intensity of bands protein was determined using an independent $t$-test. ${ }^{*} P<0.05$, compared to normal groups. Abbreviation: OV - Opisthorchis viverrini.

\section{Overall features of bile canalicular changes in O. viverrini infection}

Table 2 shows the summary of the alterations of BC features, bile volume and bile secretion-related genes caused by $O$. viverrini infection. At one month post-infection, dilatation of canalicular lumen, decrease of microvilli density and the decrease of CD10 expressions by immunohistochemical staining and western blotting 


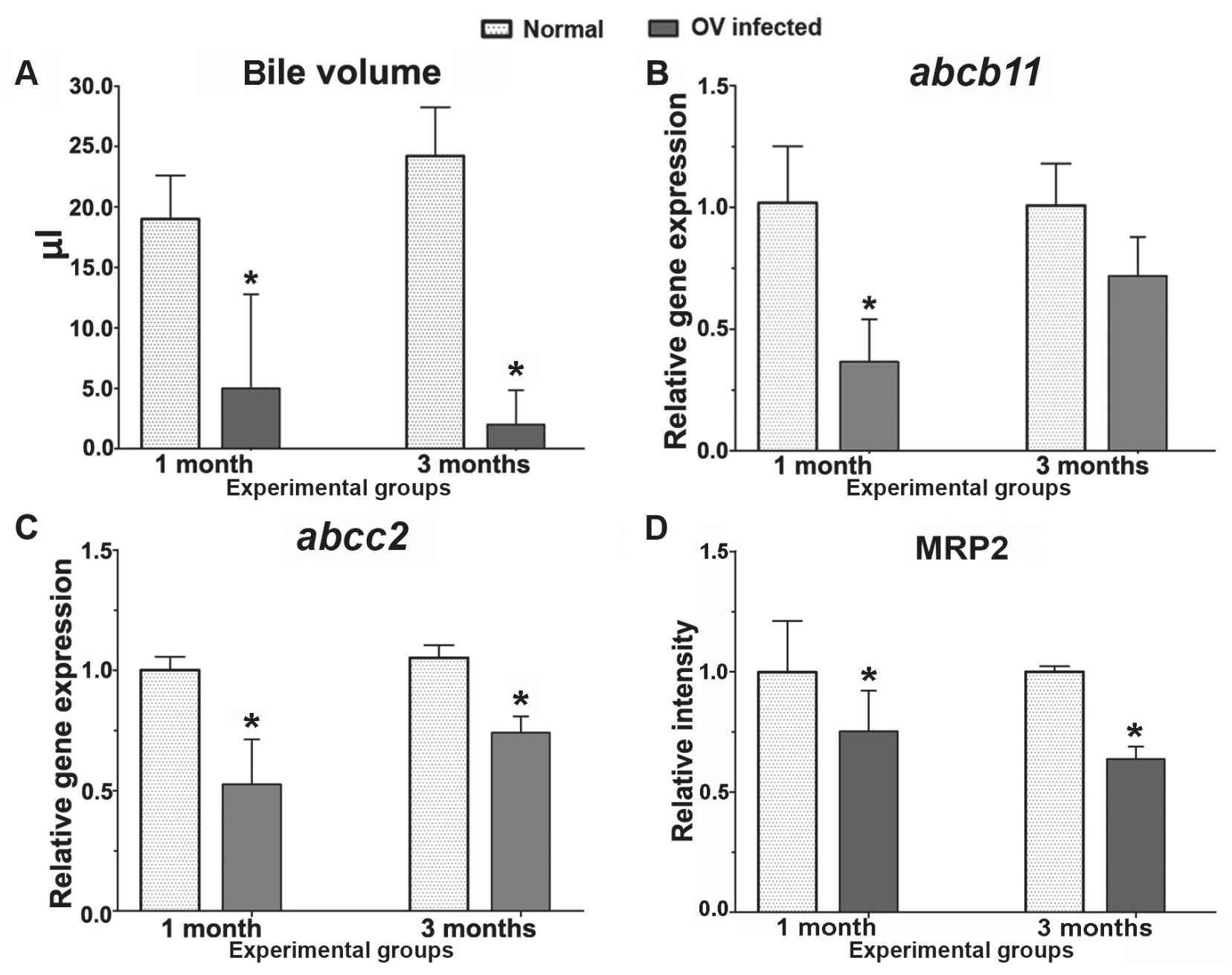

Fig. 4. Bile volume, bile acid-dependent and -independent bile flow in Opisthorchis viverrini-infected hamsters. A - bile volume $(\mu 1)$; B - bile acid-dependent bile flow ( $a b c b 11)$; C - bile acid-independent bile flow (abcc2) relative to glyceraldehyde 3-phosphate dehydrogenase (gapdh) measured by real-time RT-PCR. D - showed relative intensity of band protein. Data are expressed as mean \pm S.D. The statistical significance was tested using independent $t$-test of six hamsters in each group. $* P<0.05$, compared to normal groups. Abbreviation: OV - Opisthorchis viverrini.

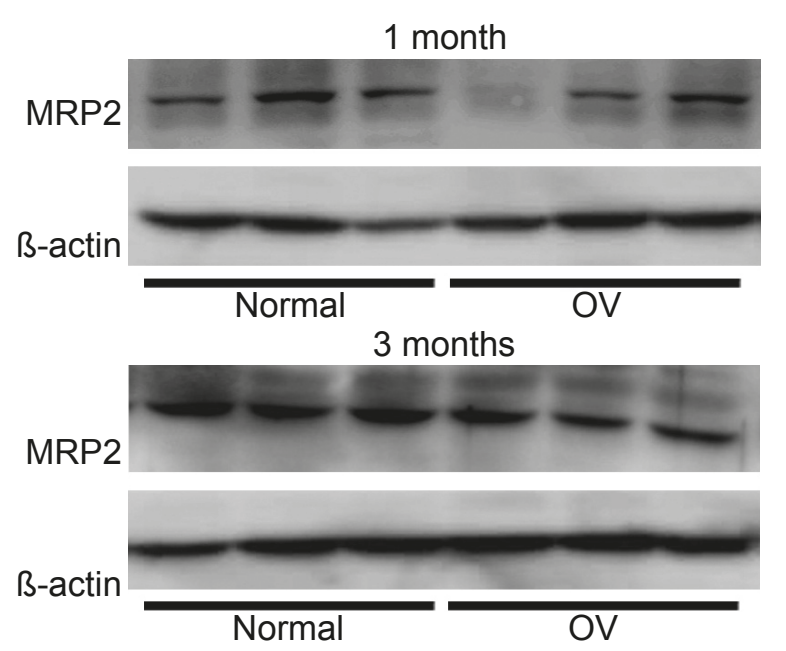

Fig. 5. $a b c c 2$ gene product; MRP2 protein expression measured by western blot. were seen in parallel to the decrease of bile volume. Expression of slc10a1, sclo1a1, abcb11, abcc2, cyp7a1 and cyp 27a1 were down-regulated, while $f x r$ and shp-1 genes was over-expressed. The expression level of MRP2 protein, a $a b c c 2$ gene product, was also down-regulated. At three months post-infection, those morphological and molecular changes of $\mathrm{BC}$ and bile-secretion associated genes or their products essentially persisted, except that $a b c b 11$ and cyp $7 a 1$ expression levels tended to return to normal level.

\section{DISCUSSION}

The present results clearly showed that infection with the liver fluke Opistorchis viverrini induced alteration of bile canaliculi (BC) and the reduction of bile production/ secretion at one and three months post-infection. Dilatation of $\mathrm{BC}$ lumen, reduction of microvilli density and 


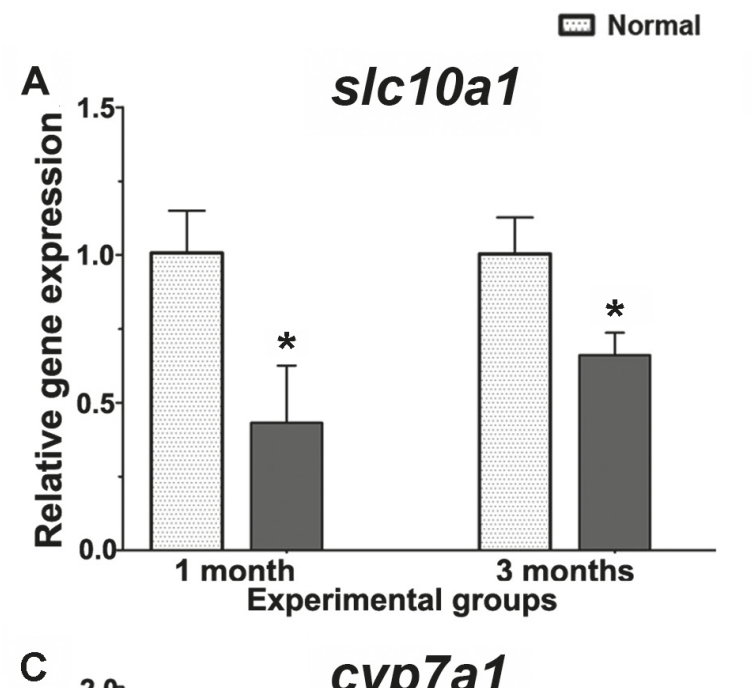

$\square O V$ infected
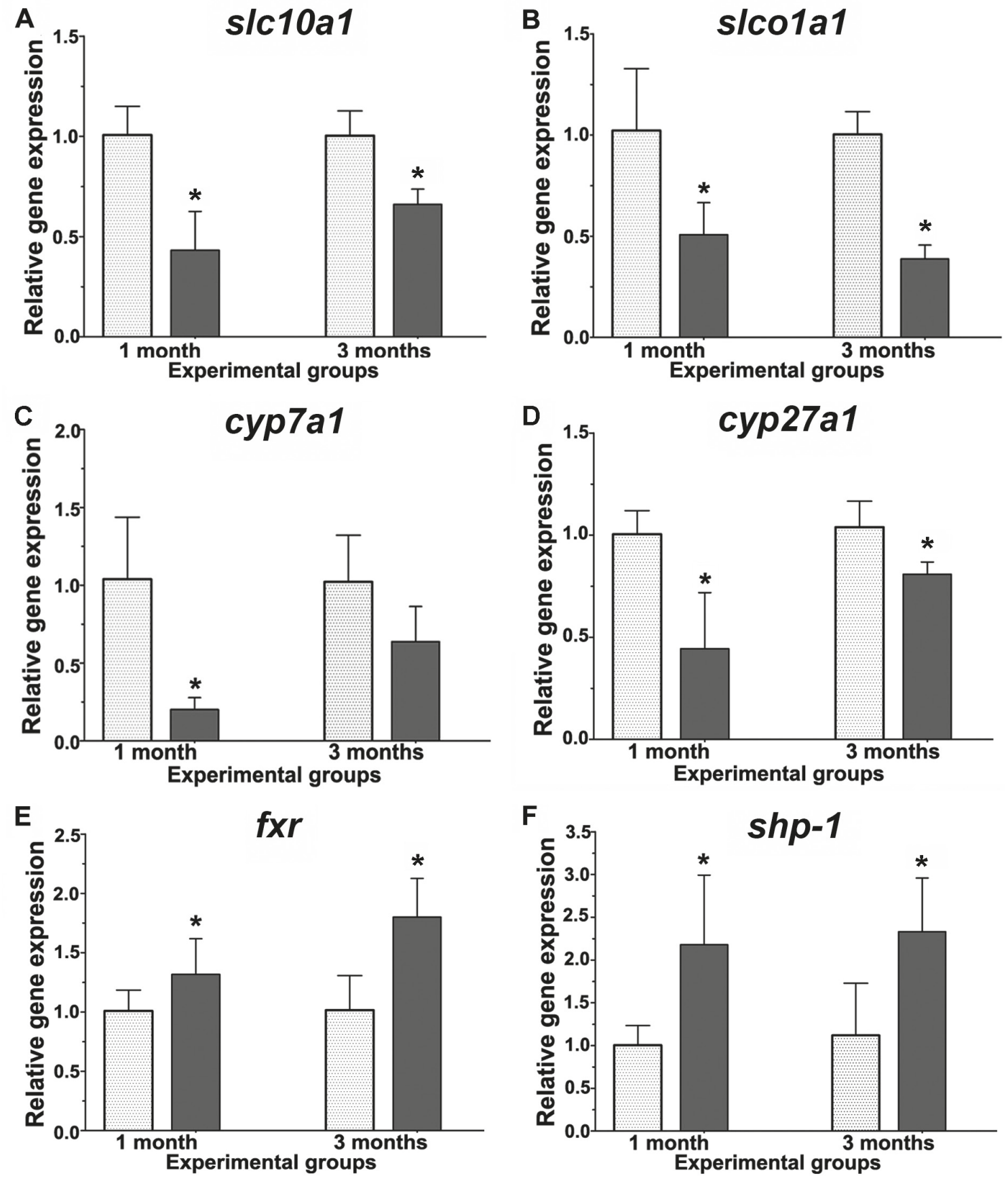

Fig. 6. Expression of bile acid uptake transporters, bile acid biosynthetic and bile acid regulatory genes in the liver of Opisthorchis viverrini-infected hamsters. A, B - mRNA levels of bile acid uptake transporters (A - slc10a1; B - slcolal); C, D - bile acid biosynthesis (C-cyp7a1; D - cyp27al); E, F - bile acid regulatory gene ( $\mathrm{E}-f x r ; \mathrm{F}-$ shp-1) relative to gapdh were evaluated by real-time RT-PCR. Data presentation and statistical analyses are the same as in Fig. $4 .{ }^{*} P<0.05$, compared to normal groups. Abbreviation: OV - Opisthorchis viverrini.

the decrease of bile volume were seen as early as at one month and persisted up to three months post-infection. After $O$. viverrini infection, parasite antigen was found in the variety of cells such as hepatocytes, bile duct epithelial cells, Kupffer cells and inflammatory cells, etc.,
(Sripa et al. 2012), which results in inflammation-mediated oxidative/nitrative stress and liver injury in hamster via free radical production (Pinlaor et al. 2004). Increase oxidative/nitrative stress is known to cause not only the hepatocyte injury (Liu et al. 2001) and BC changes (Tsai 
Table 2. Alteration of features of bile canaliculi, bile volume and bile secretory-related molecules.

\begin{tabular}{lcc}
\hline Parameters & one month & three months \\
\hline BC morphology & & \\
Width of BC (SEM) & $\uparrow$ & $\uparrow$ \\
Microvilli density (TEM) & $\downarrow$ & $\downarrow$ \\
Bile canaliculi pattern & $\downarrow$ & $\downarrow$ \\
Bile volume & $\downarrow$ & $\downarrow$ \\
Bile secretory-related molecules & & \\
Protein expression & & \\
CD10 & $\downarrow$ & $\downarrow$ \\
MRP2 & $\downarrow$ & $\downarrow$ \\
mRNA expression & & \\
slc10a1 & $\downarrow$ & $\downarrow$ \\
sclo1a1 & $\downarrow$ & $\downarrow$ \\
abcb11 & $\downarrow$ & $\leftrightarrow$ \\
abcc2 & $\downarrow$ & $\downarrow$ \\
cyp7a1 & & \\
cyp27al & $\downarrow$ & $\leftrightarrow$ \\
fxr & $\downarrow$ & $\downarrow$ \\
shp-1 & $\downarrow$ & $\uparrow$ \\
\hline
\end{tabular}

$\leftrightarrow$ unchanged when compared with normal groups; $\uparrow$ increased when compared with normal groups; $\downarrow$ decreased when compared with normal groups; $\mathrm{BC}$ - bile canaliculi.

et al. 1998), but also suppress bile production (Roma and Sanchez Pozzi 2008). Alternatively, defects in canalicular dilation and loss of canalicular network may induce canalicular contractions and contribute to decrease of bile flow (Watanabe et al. 1991) during $O$. viverrini infection.

Due to relatively large body size, adult worms in the bile duct lumen obstruct bile flow resulting in induction of $\mathrm{BC}$ alteration and decrease of bile secretion (Wonkchalee et al. 2012). After bile duct obstruction, bile reflux caused changes of the bile components and enhances BC alteration (Takakuwa et al. 2002) and damaging bile duct epithelium (Meerman et al. 1999). Taken together, inflammation-mediated oxidative/nitrative stress triggered by $O$. viverrini infection and mechanical obstruction of the bile duct lumen by adult worms induces BC alteration, decrease of bile production, defect of bile flow and change of bile components. Our findings in hamster model is relevant to the common bile duct ligation induced $\mathrm{BC}$ alteration in rat model (Vital et al. 1982).

In the present study, morphological changes of $\mathrm{BC}$ after $O$. viverrini infection paralleled to the altered expression of the genes of bile secretory-related molecules. For instance, expression of CD10, which is localised on BC surface (Loke et al. 1990) and known as the BC pattern marker, was decreased as early as at one month and persisted up to three months post-infection. A reduction of CD10 expression was associated with down-regulation of bile secretory-related genes, suggesting that CD10 might regulate the signaling pathways of these genes. Similar to our results, CD10 was diminished with $\mathrm{BC}$ defect in cholestasis and Alagille syndrome patients (Byrne et al. 2007). Furthermore, decreased expression of CD10 was found in concomitant with decrease of $a b c b 11$ and $a b c c 2$ genes, loss of density of microvilli and reduction of bile volume. Down-regulated expression of genes that control bile flow such as $a b c b 11$ may be involved in reduction of bile volume (Wang et al. 2013).

Moreover, $a b c c 2$ gene product, MRP2 protein, in the $\mathrm{BC}$ membrane is important for the elimination of many drugs and their metabolites. Down-regulation of MRP2 molecule was observed in impaired biliary excretion in intrahepatic and obstructive cholestasis (Trauner et al. 1997) and endotoxin-induced cholestasis rats (Vos et al. 1998). Down-regulation of MRP2 in our result was similar to that seen in the bile duct-obstructed rats and the chemical-induced hepatobiliary injury (Trauner et al. 1997) and in xenobiotic-induced sclerosing cholangitis and biliary fibrosis in mice (Fickert et al. 2007). In addition, we also found that the expression of MRP2 was related to alter microvilli density (Figs. 4,5 and Table 1), suggesting its possible role in bile secretion and transport (Cruz et al. 2010). Taken all those findings together, alteration of canalicular membrane and reduction of the number of microvilli (Kurbegov et al. 2003) may lead to the decrease of CD10, MRP2 and impaired canalicular secretion (Hyogo et al. 2000).

Hepatic uptake and biliary excretion of organic anions (e.g. bile acids and bilirubin) is mediated by hepatobiliary transport systems. Defects in transporter expression and function can cause or maintain cholestasis and jaundice (Geier et al. 2007). In this study, expression of the genes related to bile acid uptake transporters (slc10al and slcola1) and bile acid biosynthesis (cyp7al and cyp27a1) was decreased at one and three months post-infection with $O$. viverrini in parallel with the decrease of the bile volume. Decreased expression of these genes is similar to those observed in the obstructive cholestasis condition (Gartung et al. 1996). In addition, $O$. viverrini infection induced the upregulation of bile acid regulatory genes ( $f x r$ and $s h p-1)$, suggesting that $f x r$ and $s h p-1$ regulate cyp $7 a 1$ expression via a negative feedback mechanism (Russell 2009).

In conclusion, $O$. viverrini infection-induced $\mathrm{BC}$ alteration, decreased expression of CD10 and reduction of bile volume in association with the decrease of the expression of the genes encoding bile acid uptake transporters, bile acid-dependent and independent bile flow, bile acid biosynthesis and increased of bile acid regulatory genes. We suggest that oxidative/nitrative stress as well as bile reflux due to mechanical obstruction of $\mathrm{BC}$ cause the changes of bile acid/bile salt composition of bile, which might induce $\mathrm{BC}$ changes and the reduced expression of bile secretory-related genes and bile volume. Decrease of bile volume in hamsters infected with $O$. viverrini might lead to bile sludge formation, which is commonly seen in patients with chronic opisthorchiasis (Mairiang et al. 2012). However, the linkage between bile composition caused by $\mathrm{BC}$ changes and $\mathrm{CCA}$ genesis requires further study. 
Acknowledgements. This work was supported by The Higher Education Research Promotion and National Research University Project of Thailand, Office of the Higher Education Commission, through the Health Cluster (SHeP-GMS), Thailand, and the invitation research fund from Faculty of Medicine (I 56323), Khon Kaen University, Thailand. We also thank Miss
Nual Anong Narkkong, Central Instrumentation Unit, Faculty of Sciences, Mahasarakham University, Mahasarakham, Thailand, Assoc.Prof. Wunnee Chaijaroonkhanarak and Miss Prapaporn Jattujan, Department of Anatomy, Faculty of Medicine, Khon Kaen University, Thailand for their help in SEM and TEM studies.

\section{REFERENCES}

Alrefai W.A., Gill R.K. 2007: Bile acid transporters: structure, function, regulation and pathophysiological implications. Pharm. Res. 24: 1803-1823.

Byrne J.A., Meara N.J., Rayner A.C., Thompson R.J., KNiSELY A.S. 2007: Lack of hepatocellular CD10 along bile canaliculi is physiologic in early childhood and persistent in Alagille syndrome. Lab. Invest. 87: 1138-1148.

Chiang J.Y. 2009: Bile acids: regulation of synthesis. J. Lipid Res. 50: 1955-1966.

Cruz L.N., Guerra M.T., Kruglov E., Mennone A., Garcia C.R., Chen J., Nathanson M.H. 2010: Regulation of multidrug resistance-associated protein 2 by calcium signaling in mouse liver. Hepatology 52: 327-337.

Eaton J.E., Talwalkar J.A., Lazaridis K.N., Gores G.J., LindoR K.D. 2013: Pathogenesis of primary sclerosing cholangitis and advances in diagnosis and management. Gastroenterology 145: 521-536.

Fickert P., Stoger U., Fuchsbichler A., Moustafa T., Marschall H. U., Weiglein A. H., Tsybrovskyy O., JaeSchke H., Zatloukal K., Denk H., Trauner M. 2007: A new xenobiotic-induced mouse model of sclerosing cholangitis and biliary fibrosis. Am. J. Pathol. 171: 525-536.

Gartung C., Ananthanarayanan M., Rahman M.A., Schuele S., Nundy S., Soroka C.J., Stolz A., Suchy F.J., BOYer J.L. 1996: Down-regulation of expression and function of the rat liver $\mathrm{Na}^{+} /$bile acid cotransporter in extrahepatic cholestasis. Gastroenterology 110: 199-209.

Geier A., Wagner M., Dietrich C.G., Trauner M. 2007 : Principles of hepatic organic anion transporter regulation during cholestasis, inflammation and liver regeneration. Biochim. Biophys. Acta 1773: 283-308.

Hyogo H., Tazuma S., Kajiyama G. 2000: Biliary excretory function is regulated by canalicular membrane fluidity associated with phospholipid fatty acyl chains in the bilayer: implications for the pathophysiology of cholestasis. J. Gastroenterol. Hepatol. 15: 887-894.

IARC 2012: A review of human carcinogens: Opisthorchis viverrini and Clonorchis sinensis. IARC Monographs on the Evaluation of Carcinogenic Risks to Humans 100B: 341-370.

Jattujan P., Pinlaor S., Charoensuk L., Arunyanart C., Welbat J.U., Chaijaroonkhanarak W. 2013: Curcumin prevents bile canalicular alterations in the liver of hamsters infected with Opisthorchis viverrini. Kor. J. Parasitol. 51: 695701.

Kurbegov A.C., Setchell K.D., Haas J.E., Mierau G.W., Narkewicz M., Bancroft J.D., Karrer F., Sokol R.J. 2003: Biliary diversion for progressive familial intrahepatic cholestasis: improved liver morphology and bile acid profile. Gastroenterology 125: 1227-1234.

Lin F., Abdallah H., Meschter S. 2004: Diagnostic utility of CD10 in differentiating hepatocellular carcinoma from metastatic carcinoma in fine-needle aspiration biopsy (FNAB) of the liver. Diagn. Cytopathol. 30: 92-97.
Liu T.Z., Lee K.T., Chern C.L., Cheng J.T., Stern A., Tsai L.Y. 2001: Free radical-triggered hepatic injury of experimental obstructive jaundice of rats involves overproduction of proinflammatory cytokines and enhanced activation of nuclear factor kappa B. Ann. Clin. Lab. Sci. 31: 383-390.

Loke S.L., Leung C.Y., Chiu K.Y., Yau W.L., Cheung K.N., MA L. 1990: Localisation of CD10 to biliary canaliculi by immunoelectron microscopical examination. J. Clin. Pathol. 43: 654-656.

Mairiang E., Laha T., Bethony J.M., Thinkhamrop B., Kaewkes S., Sithithaworn P., Tesana S., Loukas A., Brindley P.J., SRiPa B. 2012: Ultrasonography assessment of hepatobiliary abnormalities in 3359 subjects with Opisthorchis viverrini infection in endemic areas of Thailand. Parasitol. Int. 61: 208-211.

Meerman L., Koopen N.R., Bloks V., Van Goor H., Havinga R., Wolthers B.G., Kramer W., Stengelin S., Muller M., Kuipers F., Jansen P.L. 1999: Biliary fibrosis associated with altered bile composition in a mouse model of erythropoietic protoporphyria. Gastroenterology 117: 696-705.

Pinlaor S., Hiraku Y., Ma N., Yongvanit P., Semba R., Oikawa S., Murata M., Sripa B., Sithithaworn P., KaWANISHI S. 2004: Mechanism of NO-mediated oxidative and nitrative DNA damage in hamsters infected with Opisthorchis viverrini: a model of inflammation-mediated carcinogenesis. Nitric Oxide 11: 175-183.

Prakobwong S., Pinlaor S., Yongvanit P., Sithithaworn P., Pairojkul C., Hiraku Y. 2009: Time profiles of the expression of metalloproteinases, tissue inhibitors of metalloproteases, cytokines and collagens in hamsters infected with Opisthorchis viverrini with special reference to peribiliary fibrosis and liver injury. Int. J. Parasitol. 39: 825-835.

Roma M.G., Sanchez Pozzi E.J. 2008: Oxidative stress: a radical way to stop making bile. An. Hepatol. 7: 16-33.

Russell D.W. 2009: Fifty years of advances in bile acid synthesis and metabolism. J. Lipid. Res. 50: S120-S125.

Serine S., Ito K., Horie T. 2006: Oxidative stress and Mrp2 internalization. Free Radic. Biol. Med. 40: 2166-2174.

Sripa B., Brindley P.J., Mulvenna J., Laha T., Smout M.J., Mairiang E., Bethony J. M., Loukas A. 2012: The tumorigenic liver fluke Opisthorchis viverrini - multiple pathways to cancer. Trends Parasitol. 28: 395-407.

Takakuma Y., Kokai Y., Sasaki K., Chiba H., Tobioka H., Mori M., SAWAda N. 2002: Bile canalicular barrier function and expression of tight-junctional molecules in rat hepatocytes during common bile duct ligation. Cell Tissue Res. 307: 181189.

Trauner M., Arrese M., Soroka C.J., Ananthanarayanan M., Koeppel T.A., Schlosser S.F., Suchy F.J., Keppler D., Boyer J.L. 1997: The rat canalicular conjugate export pump (Mrp2) is down-regulated in intrahepatic and obstructive cholestasis. Gastroenterology 113: 255-264. 
Trauner M., Boyer J.L. 2003: Bile salt transporters: molecular characterization, function, and regulation. Physiol. Rev. 83: 633-671.

Tsai L.Y., Lee K.T., LiU T.Z. 1998: Evidence for accelerated generation of hydroxyl radicals in experimental obstructive jaundice of rats. Free Radic. Biol. Med. 24: 732-737.

Tyson G.L., El-Serag H.B. 2011: Risk factors for cholangiocarcinoma. Hepatology 54: 173-184.

Vital A., Bioulac-Sage P., Iron A., Balabaud C. 1982: Morphologic structure of bile canaliculi after bile duct ligation in the rat. A time-course study. Arch Pathol. Lab Med. 106: $464-467$.

Vos T.A., Hooiveld G.J., Koning H., Childs S., Meijer D.K., Moshage H., Jansen P.L., Muller M. 1998: Up-regulation of the multidrug resistance genes, Mrp1 and Mdrlb, and downregulation of the organic anion transporter, Mrp2, and the bile salt transporter, Spgp, in endotoxemic rat liver. Hepatology 28: $1637-1644$.

Wang R., Liu L., Sheps J. A., Forrest D., Hofmann A.F., Hagey L.R., Ling V. 2013: Defective canalicular transport and toxicity of dietary ursodeoxycholic acid in the abcb11 1 -

Received 29 April 2014 mouse: transport and gene expression studies. Am. J. Physiol. Gastrointest. Liver Physiol. 305: G286-294.

Watanabe N., Tsukada N., Smith C.R., Phillips M.J. 1991: Motility of bile canaliculi in the living animal: implications for bile flow. J. Cell Biol. 113: 1069-1080.

Wonkchalee O., Boonmars T., Kaewkes S., Chamgramol Y., Aromdee C., Wu Z., Juasook A., Sudsarn P., BoonJaraspinyo S., Pairojkul C. 2012: Comparative studies on animal models for Opisthorchis viverrini infection: host interaction through susceptibility and pathology. Parasitol. Res. 110: 1213-1223.

Yamamoto K., Itoshima T., Tsuji T., Murakami T. 1990: Three-dimensional fine structure of the biliary tract: scanning electron microscopy of biliary casts. J. Electron Microsc. Tech. 14: 208-217.

Yeh T.H., Krauland L., Singh V., Zou B., Devaraj P., Stolz D.B., Franks J., Monga S.P., Sasatomi E., Behari J. 2010: Liver-specific beta-catenin knockout mice have bile canalicular abnormalities, bile secretory defect, and intrahepatic cholestasis. Hepatology 52: 1410-1419. 